\title{
Electrical plasmon detection in graphene waveguides
}

\author{
Iacopo Torre, ${ }^{1}$ Andrea Tomadin, ${ }^{1}$ Roman Krahne, ${ }^{2}$ Vittorio Pellegrini, ${ }^{3,1}$ and Marco Polini ${ }^{1,3, *}$ \\ ${ }^{1}$ NEST, Istituto Nanoscienze-CNR and Scuola Normale Superiore, I-56126 Pisa, Italy \\ ${ }^{2}$ Istituto Italiano di Tecnologia, Graphene Labs and Nanochemistry Department, Via Morego 30, I-16163 Genova, Italy \\ ${ }^{3}$ Istituto Italiano di Tecnologia, Graphene Labs, Via Morego 30, I-16163 Genova, Italy
}

(Received 17 October 2014; revised manuscript received 28 November 2014; published 5 February 2015)

\begin{abstract}
We present a simple device architecture that allows all-electrical detection of plasmons in a graphene waveguide. The key principle of our electrical plasmon detection scheme is the nonlinear nature of the hydrodynamic equations of motion that describe transport in graphene at room temperature and in a wide range of carrier densities. These nonlinearities yield a dc voltage in response to the oscillating field of a propagating plasmon. For illustrative purposes, we calculate the dc voltage arising from the propagation of the lowest-energy modes in a fully analytical fashion. Our device architecture for all-electrical plasmon detection paves the way for the integration of graphene plasmonic waveguides in electronic circuits.
\end{abstract}

PACS number(s): 73.20.Mf, 72.80.Vp

Introduction. The two-dimensional (2D) electron liquid in a doped graphene sheet [1] supports plasmons with energies from the far infrared to the visible, depending on carrier concentration [2]. Although they share similarities with plasmons in ordinary parabolic-band 2D electron liquids [3], plasmons in graphene are profoundly different. From a fundamental point of view, their dispersion relation is sensitive to many-body effects even in the long-wavelength limit [4]. More practically, plasmons in graphene are easily accessible to surface-science probes and optoelectrical manipulation since they are exposed and not buried in a quantum well.

Plasmons in graphene are also substantially different from those in noble metals. Indeed, recent near-field optical spectroscopy experiments [5-9] have demonstrated that plasmons in graphene display gate tunability and ultrastrong field confinement. Moreover, low damping rates can be achieved by employing graphene samples encapsulated in hexagonal boron nitride thin slabs [9-12]. For these reasons, graphene plasmonics has recently attracted a great deal of interest [13]. Graphene plasmons may allow for new classes of devices for single-plasmon nonlinearities [14], extraordinarily strong light-matter interactions [15], deep subwavelength metamaterials [16-19], and photodetectors with enhanced sensitivity $[20,21]$.

A key ingredient of a disruptive plasmonic platform is the ability to efficiently detect plasmons in all-electrical manners. Some progress has been made in this direction in conventional noble-metal-based plasmonics. Falk et al. [22], for example, were able to couple plasmons in Ag nanowires to nanowire Ge field-effect transistors. Built-in electric fields in the latter are used to separate electrons and holes before recombination, thereby giving rise to a measurable sourcedrain current. Similarly, Neutens et al. [23] employed an integrated metal-semiconductor-metal detector in a metalinsulator-metal plasmon waveguide.

While graphene plasmons have been detected and studied in a multitude of ways [13], including electron energy loss spectroscopy [24], polarized Fourier transform infrared spectroscopy [16], and near-field optical spectroscopy [5-9], a protocol for all-electrical detection of these modes is still lacking.

\footnotetext{
*m.polini@sns.it
}

In this Rapid Communication we present a device architecture that allows all-electrical detection of plasmons in graphene waveguides. In our scheme, all-electrical detection is not enabled by the integration of a detector in a graphene plasmon waveguide (GPW) but rather by the intrinsic nonlinear terms in the hydrodynamic equations that describe transport in the 2D massless Dirac fermion (MDF) liquid [1] hosted by graphene. Nonlinearities enable the emergence of a rectified (i.e., dc) component $\delta V(\boldsymbol{r})$ of the ac electric field of a propagating plasmon, which can be measured by a suitable geometry of ohmic contacts placed along the GPW, as shown in Fig. 1. We now present a calculation of the spatially dependent electrical signal $\delta V(\boldsymbol{r})$.

Hydrodynamic theory. We consider a GPW with transverse (longitudinal) size $W$ ( $L$ with $L \gg W$ ), which is embedded between two insulators with dielectric constants $\epsilon_{1}$ (above the GPW) and $\epsilon_{2}$ (below the GPW). Here, "longitudinal" and "transverse" refer to the plasmon propagation direction- $\hat{\boldsymbol{x}}$ in Fig. 1.

We would like to describe ac transport in a GPW by employing the theory of hydrodynamics [25]. We therefore need to assess whether experimentally relevant regions of parameter space exist in which this theory is applicable. First, at room temperature and for typical carrier densities $(\bar{n} \simeq$ $10^{11} \mathrm{~cm}^{-2}-5 \times 10^{12} \mathrm{~cm}^{-2}$ ), the mean free path $\ell_{\mathrm{ee}}=v_{\mathrm{F}} \tau_{\mathrm{ee}}$ for electron-electron collisions in graphene is short [26,27], i.e., $\ell_{\mathrm{ee}} \simeq 100-150 \mathrm{~nm}$. Here, $v_{\mathrm{F}} \simeq 10^{6} \mathrm{~m} / \mathrm{s}$ is the graphene Fermi velocity [28] and $\tau_{\mathrm{ee}} \simeq 100 \mathrm{fs}=10^{-13} \mathrm{~s}$ is the electronelectron collision time $[26,27]$. Second, for hydrodynamics to provide a correct description of the response of the system at finite frequencies, it must also be $\omega \tau_{\mathrm{ee}} \ll 1$, where $\omega$ is the external-excitation angular frequency. The value of $\tau_{\text {ee }}$ given above constrains the maximum external-excitation frequency to be $f_{\max } \equiv 1 /\left(2 \pi \tau_{\mathrm{ee}}\right) \lesssim 3 \mathrm{THz}$. We therefore conclude that, for $\bar{n} \simeq 10^{11} \mathrm{~cm}^{-2}-5 \times 10^{12} \mathrm{~cm}^{-2}, \omega<2 \pi f_{\max }$, and $T=$ $300 \mathrm{~K}$, transport in GPWs with characteristic dimensions $L, W \gg \ell_{\mathrm{ee}}$ is accurately described by hydrodynamic equations of motion [25]. Related continuum-model descriptions of plasmons in GPWs have been employed in Refs. [29-32].

The set of hydrodynamic equations consists of (i) the continuity equation,

$$
\partial_{t} n(\boldsymbol{r}, t)+\nabla \cdot[n(\boldsymbol{r}, t) \boldsymbol{v}(\boldsymbol{r}, t)]=\mathbf{0},
$$




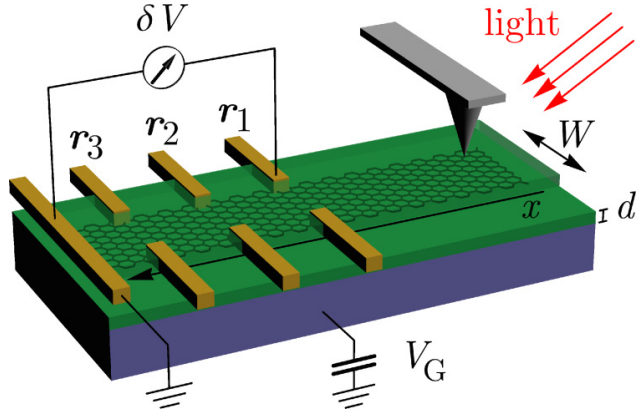

FIG. 1. (Color online) Schematics of our electrical plasmon detector. A graphene strip of width $W$ is encapsulated between two dielectrics (semitransparent slab above and dark green slab underneath graphene). A back gate (dark blue slab), separated by a distance $d$ from the graphene sheet and held at a voltage $V_{\mathrm{G}}$, is used to control the average carrier density $\bar{n}$ in graphene. At one end of the strip, a plasmon is launched by using, e.g., a metallized atomic force microscope tip illuminated by light [5-9]. Due to nonlinearities in the hydrodynamic equations, a dc electrical potential difference $\delta V$ is measured between probe electrodes placed at positions $\boldsymbol{r}_{1}, \boldsymbol{r}_{2}$, and $\boldsymbol{r}_{3}$ and a reference electrode placed at the other end of the strip. The quantity $\delta V$ provides a direct measurement of the ac electric field of a propagating plasmon.

and (ii) the Navier-Stokes equation [25]

$$
m_{\mathrm{c}} n(\boldsymbol{r}, t) D_{t} \boldsymbol{v}(\boldsymbol{r}, t)=-e n(\boldsymbol{r}, t) \boldsymbol{E}(\boldsymbol{r}, t)+\eta \nabla^{2} \boldsymbol{v}(\boldsymbol{r}, t) .
$$

In Eqs. (1) and (2), $n(\boldsymbol{r}, t)$ is the carrier density and $\boldsymbol{v}(\boldsymbol{r}, t)$ is the drift velocity. In Eq. (2), $m_{\mathrm{c}}=\hbar \sqrt{\pi \bar{n}} / v_{\mathrm{F}}$ is the graphene cyclotron mass [28], with $\bar{n}=C V_{\mathrm{G}} / e$ the average electron density and $V_{\mathrm{G}}$ the back-gate voltage (see Fig. 1), and $D_{t} \equiv$ $\partial_{t}+\boldsymbol{v}(\boldsymbol{r}, t) \cdot \nabla$ is the convective derivative [25]. The electric field $\boldsymbol{E}(\boldsymbol{r}, t)=-\nabla \Phi(\boldsymbol{r}, t)$ is the gradient of the electrostatic potential $\Phi(\boldsymbol{r}, t)$ (we neglect retardation effects). Finally, $\eta$ is the shear viscosity of the 2D electron liquid [3,25]. For future purposes, we also introduce the kinematic viscosity [25]

$$
v \equiv \frac{\eta}{\bar{n} m_{\mathrm{c}}} .
$$

It can be shown [33] that, in the hydrodynamic $\omega \tau_{\mathrm{ee}} \ll 1$ limit, $v \simeq v_{\mathrm{F}}^{2} \tau_{\mathrm{ee}} / 4$. With the values of $v_{\mathrm{F}}$ and $\tau_{\mathrm{ee}}$ given above, we find $v \simeq 250 \mathrm{~cm}^{2} / \mathrm{s}$. In writing Eq. (2) we have neglected a term due to the bulk viscosity $\zeta$ since this quantity vanishes at long wavelengths $[3,25]$.

We highlight two nonlinear terms in Eqs. (1) and (2): (a) the nonlinear coupling between $n(\boldsymbol{r}, t)$ and $\boldsymbol{v}(\boldsymbol{r}, t)$, which is present in Eq. (1), and (b) the nonlinear term $[\boldsymbol{v}(\boldsymbol{r}, t) \cdot \nabla] \boldsymbol{v}(\boldsymbol{r}, t)$ in Eq. (2), representing the convective acceleration [25].

Momentum-nonconserving collisions, such as those due to the friction of the electron liquid against the disorder potential, can be taken into account phenomenologically by adding a term of the type $-m_{\mathrm{c}} \gamma n(\boldsymbol{r}, t) \boldsymbol{v}(\boldsymbol{r}, t)$ on the right-hand side of Eq. (2), where $\gamma$ is a damping rate [34]. Furthermore, corrections to Eq. (2), stemming from the pseudorelativistic nature of MDF flow in graphene, can be easily incorporated into the theory $[35,36]$ and have been demonstrated to yield stronger rectified signals [35].

Finally, to close the set of equations, we need a relation between $\Phi(\boldsymbol{r}, t)$ and $n(\boldsymbol{r}, t)$. This depends on the screening exerted by dielectrics and conductors near the GPW. If a metal gate is positioned underneath the GPW at a distance $d \ll$ $W, k^{-1}$, where $k$ is the plasmon wave vector, the following local relation exists [35]:

$$
\Phi(\boldsymbol{r}, t) \approx-\frac{e}{C} \delta n(\boldsymbol{r}, t),
$$

where $C=\epsilon_{2} /(4 \pi d)$ is a capacitance per unit area and $\delta n(\boldsymbol{r}, t) \equiv n(\boldsymbol{r}, t)-\bar{n}$. Equation (4) greatly simplifies the theoretical analysis and, in fact, allows us to solve the problem in a fully analytical fashion [37], as we now detail.

Equations (1)-(4) need to be accompanied by boundary conditions. As explained in Sec. I of Ref. [38] and in Ref. [39], we fix $v_{y}(x, y=0, W)=0$ and $\partial_{x} v_{y}(x, y=0, W)+$ $\partial_{y} v_{x}(x, y=0, W)=0$.

Linear response theory and plasmons. The GPW supports collective charge density oscillations, i.e., plasmons [3], which propagate along the $\hat{\boldsymbol{x}}$ direction and are confined in the $\hat{\boldsymbol{y}}$ direction. To calculate the frequency spectrum and potential profiles of these modes we have to linearize Eqs. (1), (2), and (4). We write $n(\boldsymbol{r}, t)=\bar{n}+n_{1}(\boldsymbol{r}, t)+$ $n_{2}(\boldsymbol{r}, t)+\ldots, \boldsymbol{v}(\boldsymbol{r}, t)=\boldsymbol{v}_{1}(\boldsymbol{r}, t)+\boldsymbol{v}_{2}(\boldsymbol{r}, t)+\ldots$, and $\Phi(\boldsymbol{r}, t)=$ $\Phi_{1}(\boldsymbol{r}, t)+\Phi_{2}(\boldsymbol{r}, t)+\ldots$. Here $n_{1}(\boldsymbol{r}, t), \boldsymbol{v}_{1}(\boldsymbol{r}, t)$, and $\Phi_{1}(\boldsymbol{r}, t)$ $\left[n_{2}(\boldsymbol{r}, t), \boldsymbol{v}_{2}(\boldsymbol{r}, t)\right.$, and $\left.\Phi_{2}(\boldsymbol{r}, t)\right]$ denote first-order (secondorder) corrections with respect to equilibrium values (by "equilibrium" we here mean the state of the GPW in which a plasmon is not propagating). In the linearized theory we retain only terms of the first order. All the relevant details are reported in Secs. II and III of Ref. [38].

For the sake of simplicity, we assume a uniform equilibrium electron density in the GPW, disregarding the well-known inhomogeneous doping $\bar{n} \rightarrow \bar{n}(y)$ that arises due to a back gate. Plasmons in back-gated waveguides, however, have been demonstrated [40] to be similar to those of uniformly doped waveguides, provided that the Fermi energy is appropriately scaled to compensate for the singular behavior of the carrier density $\bar{n}(y)$ as $y \rightarrow 0, W$.

Plasmon modes are labeled by a wave number $k$ (stemming from translational invariance along the $\hat{\boldsymbol{x}}$ direction) and a discrete index $n=0,1,2, \ldots$ The associated ac electrical potential is given by

$$
\Phi_{1}(\boldsymbol{r}, t)=\varphi_{n}(y) e^{i k x-i \omega_{n}(k) t},
$$

where

$$
\varphi_{n}(y)=\frac{1}{\sqrt{W}} \begin{cases}1, & \text { for } n=0, \\ \sqrt{2} \cos [n \pi y / W], & \text { for } n \neq 0 .\end{cases}
$$

The mode dispersion reads as

$$
\omega_{n}(k)=\sqrt{s^{2} K_{n}^{2}-\frac{\left(\gamma+v K_{n}^{2}\right)^{2}}{4}}-i \frac{\gamma+v K_{n}^{2}}{2},
$$

where $K_{n}^{2}=k^{2}+q_{n}^{2}, q_{n} \equiv \pi n / W$, and $s=\sqrt{e^{2} \bar{n} /\left(C m_{\mathrm{c}}\right)}$ is the hydrodynamic speed of sound. It is useful to introduce the following natural frequency scale: $\Omega_{0} \equiv s / W$. Setting external $(\gamma)$ and internal $(v)$ dissipation to zero in Eq. (7) we find the expected result, $\omega_{n}(k)=s K_{n}$. The lowest-energy $n=0$ mode shows an acoustic dispersion due to screening by the back gate. Modes with $n \neq 0$ are gapped, i.e., $\omega_{n}(k \rightarrow 0)=n \pi \Omega_{0}$. The fundamental frequency is $\omega_{n=1} /(2 \pi)=\Omega_{0} / 2 \simeq 1.0 \mathrm{THz}$ 


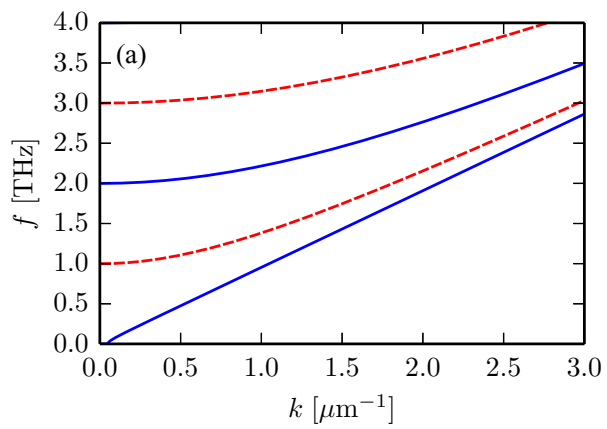

general grounds [42], we expect that the second-order response is composed by an oscillating component at frequency $2 \omega$ (i.e., second-harmonic generation) and a steady component. Since we are interested in detecting a dc signal, we can extract the rectified voltage $\delta V(\boldsymbol{r})$ from the time average over one period of the external radiation of the second-order potential fluctuations: $\delta V(\boldsymbol{r}) \equiv\left\langle\Phi_{2}(\boldsymbol{r}, t)\right\rangle$. Averaging over time the second-order equations we obtain [38]

$$
\nabla \cdot \delta \boldsymbol{v}(\boldsymbol{r})=-\frac{1}{\bar{n}} \nabla \cdot\left\langle n_{1}(\boldsymbol{r}, t) \boldsymbol{v}_{1}(\boldsymbol{r}, t)\right\rangle
$$
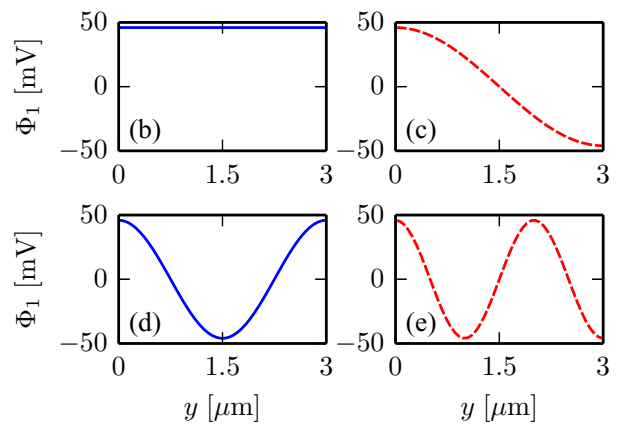

FIG. 2. (Color online) (a) Dispersion relation $f_{n}(k) \equiv$ $\omega_{n}(k) /(2 \pi)$ of four low-energy plasmon modes $(n=0, \ldots, 3)$ in a GPW with the following parameters: $W=3 \mu \mathrm{m}, d=100 \mathrm{~nm}$, $\epsilon_{2}=3.9, \quad \bar{n}=10^{12} \mathrm{~cm}^{-2}, \quad \gamma=0.3 \Omega_{0}, \quad$ and $\quad v=250 \mathrm{~cm}^{2} / \mathrm{s}$. Solid (dashed) lines correspond to even (odd) modes. (b)-(e) Corresponding electrical potential profiles $\Phi_{1}(\boldsymbol{r}, t)$ evaluated at $x=0$ and $t=0$ and plotted as functions of the transverse $y \in[0, W]$ coordinate. These results have been obtained by imposing electron density fluctuations equal to [41] $\delta n / \bar{n}=1 \%$.

for $W=3 \mu \mathrm{m}, d=100 \mathrm{~nm}, \epsilon_{2}=3.9$, and $\bar{n}=10^{12} \mathrm{~cm}^{-2}$. Dispersion relations and mode profiles for the above set of parameters are shown in Fig. 2. In the approximation (4) the results do not depend on $\epsilon_{1}$.

When the $n$th eigenmode of the GPW is excited by an external perturbation with frequency $\omega$, it propagates with a complex wave number,

$$
k_{n}(\omega)=\sqrt{\frac{\omega^{2}+i \omega \gamma}{s^{2}-i \nu \omega}-q_{n}^{2}} .
$$

The wave number, $\operatorname{Re}\left(k_{n}\right)$, and inverse damping ratio, $\gamma_{n}^{-1} \equiv \operatorname{Re}\left(k_{n}\right) / \operatorname{Im}\left(k_{n}\right)$, of the launched plasmon depend only on the excitation frequency $\omega$ and not on details of the tipsample coupling [5-9]. Physically, the dimensionless number $\gamma_{n}^{-1}$ controls the plasmon extinction length $\ell_{n} \equiv 1 / \operatorname{Im}\left(k_{n}\right)=$ $\gamma_{n}^{-1} \lambda_{n} /(2 \pi)$, with $\lambda_{n}=2 \pi / \operatorname{Re}\left(k_{n}\right)$ the plasmon wavelength. With the value of $v$ given above and $\gamma=0.3 \Omega_{0}$, the inverse damping ratio of the $n=0$ mode is $\gamma_{0}^{-1} \simeq 23$, while $\gamma_{1}^{-1} \simeq 7$ for $n=1$.

Second-order theory of all-electrical detection. The rectified signal can be calculated by keeping track of the secondorder terms $n_{2}(\boldsymbol{r}, t), \boldsymbol{v}_{2}(\boldsymbol{r}, t)$, and $\Phi_{2}(\boldsymbol{r}, t)$ in the expansion of the hydrodynamic variables. Physically, the second-order response describes interactions between propagating modes. If only one mode propagates, a dc signal due to self-mixing of the plasmon field is generated. If more than one mode propagates, also interference terms will be generated. On

and

$$
\begin{aligned}
&- \frac{e}{m_{\mathrm{c}}} \nabla \delta V(\boldsymbol{r})+\gamma \delta \boldsymbol{v}(\boldsymbol{r})-v \nabla^{2} \delta \boldsymbol{v}(\boldsymbol{r}) \\
&=-\epsilon\left\langle\left[\boldsymbol{v}_{1}(\boldsymbol{r}, t) \cdot \nabla\right] \boldsymbol{v}_{1}(\boldsymbol{r}, t)\right\rangle-\frac{v}{\bar{n}}\left\langle n_{1}(\boldsymbol{r}, t) \nabla^{2} \boldsymbol{v}_{1}(\boldsymbol{r}, t)\right\rangle,
\end{aligned}
$$

where $\delta \boldsymbol{v} \equiv\left\langle\boldsymbol{v}_{2}(\boldsymbol{r}, t)\right\rangle$ is the time average of the velocity fluctuations. We urge the reader to note that in Eq. (10) we have introduced a dimensionless parameter $\epsilon$ which allows us to keep track of the role of different nonlinearities in determining the rectified signal. By setting $\epsilon=0$ one neglects the convective nonlinearity in the Navier-Stokes equation. Moreover, by setting $\epsilon=0$ and $v=0$, the Navier-Stokes equation reduces to the linearized Euler equation [25], which leads to the standard Drude formula for the local conductivity. However, a finite rectified signal $\delta V(\boldsymbol{r})$ exists in this case, too, and is entirely due to the nonlinear $n(\boldsymbol{r}, t) \boldsymbol{v}(\boldsymbol{r}, t)$ coupling in the continuity equation.

Equations (9) and (10) are crucial since they relate the second-order quantities $\delta V$ and $\delta \boldsymbol{v}$ to the quantities $n_{1}(\boldsymbol{r}, t)$ and $\boldsymbol{v}_{1}(\boldsymbol{r}, t)$, which have been calculated in the linearized theory. Furthermore, they can be used to calculate the dc signal $\delta V(\boldsymbol{r})$ in response to plasmon propagation in any desired geometry. As stated above, $\delta V(\boldsymbol{r})$ can be measured by employing a set of ohmic contacts as in Fig. 1.

We now evaluate $\delta V(\boldsymbol{r})$ for the experimentally relevant case in which plasmons are launched at a specific location $\boldsymbol{r}^{\star}=\left(0, y^{\star}\right)$ with $y^{\star} \in[0, W]$ in the GPW. The quantity $\delta V(\boldsymbol{r})$ for $x \gg W$ and arbitrary $y$ can then be calculated according to the following procedure, which is typical of a scattering problem. (a) For $x \gg W$ the plasmon velocity field can be written as a sum over propagating modes [i.e., modes with $\omega_{n}(k)<\omega$, where $\omega$ is the angular frequency of the stimulus that launches plasmons]. All the other modes, which can be excited near $\boldsymbol{r}^{\star}$, exponentially damp out at large distances since they have a purely imaginary $k$-see Eq. (8). Furthermore, as shown in Sec. III of Ref. [38], the plasmon velocity field $\boldsymbol{v}_{1}$ is irrotational at large distances, i.e., $\boldsymbol{\nabla} \times \boldsymbol{v}_{1}=\mathbf{0}$ for $x \gg W$. Since $\nabla \times \nabla \phi(r) \equiv \mathbf{0}$ for an arbitrary scalar function $\phi(\boldsymbol{r})$, we conclude that $\boldsymbol{v}_{1}(\boldsymbol{r}, t)$ for $x \gg W$ can be written as the gradient of a scalar function. In the language of scattering theory, we have built the so-called asymptotic solution, which we denote by $\boldsymbol{v}_{1}^{(\mathrm{a})}(\boldsymbol{r}, t)$. (b) Let us imagine that an external perturbation with frequency $\omega$ launches, for example, an arbitrary linear combination with complex coefficients of the $n=0$ and $n=1$ GPW modes. Because of (a), we can write the corresponding 

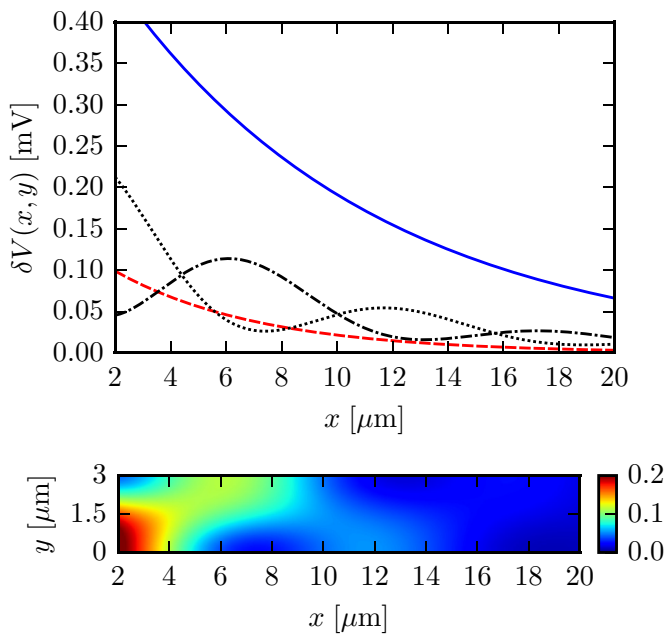

FIG. 3. (Color online) (a) The dc potential $\delta V(\boldsymbol{r})$ (in millivolts) as a function of $x$ (in $\mu \mathrm{m}$ ), calculated at the edges of the GPW, i.e., at $y=0$ (solid, dashed, and dotted lines) and $y=W$ (dashed-dotted line). These numerical results have been calculated by setting the following parameter values in Eq. (11): $\xi=0$ and $\alpha=0$ (solid line), $\xi=1$ and $\alpha=0$ (dashed line), $\xi=1 / 2$ and $\alpha=\pi / 4$ (dotted and dashed-dotted lines). The other parameters are as in Fig. 2. Finally, we have taken $\omega /(2 \pi) \simeq 1.2 \mathrm{THz}$ in Eq. (8). Note that $\omega /(2 \pi)$ is $20 \%$ larger than the fundamental frequency for the laser to be able to excite the two lowest modes of the GPW. The scale is in millivolts. In all cases the value of $A$ has been chosen to yield $\delta n / \bar{n}=1 \%$. This normalization implies different values of $A$ for different values of $\xi, \alpha$. (b) Spatial map of the dc potential $\delta V(\boldsymbol{r})$ calculated by setting $\xi=1 / 2$ and $\alpha=\pi / 4$. The color bar shows the potential amplitude in millivolts. All other parameters are as in (a).

asymptotic velocity field as

$$
\begin{aligned}
\boldsymbol{v}_{1}^{(\mathrm{a})}(\boldsymbol{r}, t)= & \frac{A}{2} \nabla\left[(1-\xi) \varphi_{0}(y) e^{i \bar{k}_{0} x} e^{-\beta_{0} x}\right. \\
& \left.+\xi e^{i \alpha} \varphi_{1}(y) e^{i \bar{k}_{1} x} e^{-\beta_{1} x}\right] e^{-i \omega t}+\text { c.c. },
\end{aligned}
$$

where $x>0$ and the functions $\varphi_{n}(y)$ have been introduced earlier in Eq. (6). In Eq. (11), $A=\bar{v} W^{3 / 2}$ is an unknown amplitude (here $\bar{v}$ has physical dimensions of a velocity), which can be estimated as discussed below, $\bar{k}_{n}=\operatorname{Re}\left[k_{n}(\omega)\right]$ and $\beta_{n}=\operatorname{Im}\left[k_{n}(\omega)\right]$ with $k_{n}(\omega)$ as in Eq. $(8), \xi \in[0,1]$ is a real parameter that allows us to interpolate between the case in which only the $n=0$ mode is launched $(\xi=0)$ and the case in which only the $n=1$ mode is launched $(\xi=1)$, and $e^{i \alpha}$ (with $\alpha$ real) is the relative phase between the two modes. In the case in which plasmons are launched at $\boldsymbol{r}^{\star}$ by using a metallized tip illuminated by light [5-9], $\xi$ depends on the tip-sample coupling: For example, for a tip placed symmetrically with respect to the GPW axis, $\xi$ vanishes. In practice, $\xi$ can be varied by moving the tip along the $\hat{\boldsymbol{y}}$ direction. (c) With the velocity field in Eq. (11), one can easily calculate the asymptotic density profile $n_{1}^{(\mathrm{a})}(\boldsymbol{r}, t)$ from the continuity equation. (d) The quantities $n_{1}^{(\mathrm{a})}(\boldsymbol{r}, t)$ and $\boldsymbol{v}_{1}^{(\mathrm{a})}(\boldsymbol{r}, t)$ are then used to calculate the temporal averages that appear on the right-hand side of Eqs. (9) and (10). (e) Finally, $\delta V(\boldsymbol{r})$ is found by solving Eqs. (9) and (10).

Simple and compact analytical expressions, obtained by following steps (a)-(e), are available for $\delta V(\boldsymbol{r})$ in the extreme cases $\xi=0$ and $\xi=1$ and are presented in Sec. IV of Ref. [38]. In the general $\xi \neq 0,1$ case, an oscillatory term with spatial periodicity $2 \pi /\left(\bar{k}_{0}-\bar{k}_{1}\right)$ appears along the $\hat{\boldsymbol{x}}$ direction due to interference of the two modes in Eq. (11). Illustrative numerical results can be found in Fig. 3. Notice that the dc signal is $\lesssim 0.5 \mathrm{mV}$ and that its spatial extension is $\sim 20 \mu \mathrm{m}$. The dc potentials on the top and bottom GPW edges are not equal in the case $\xi \neq 0,1$ since Eq. (11) is a superposition of modes with different parity. The quantity $A$ in Eq. (11) was estimated with reference to Refs. [5-9], where a metallized tip is used to launch plasmons. In this case, $A$ can be calculated starting from the amplitude of density oscillations $\delta n$ in units of $\bar{n}$, created by the tip at $\boldsymbol{r}^{\star}$. The results in Fig. 3 have been produced by using [41] $\delta n / \bar{n}=1 \%$ at $x=0$. This "normalization" condition yields different values of $A$ for different values of $\xi, \alpha$. In other words, $A$ measures how well the tip couples to the linear combination of modes in Eq. (11). The $\xi=0$ mode has a better coupling to the tip (and therefore yields a larger dc signal) than the linear superposition of $n=0$ and $n=1$ modes corresponding to $\xi=1 / 2$ and $\alpha=\pi / 4$.

Conclusions. In summary, we have discussed an architecture based on a graphene waveguide where electrical detection of plasmons may be experimentally achieved. We have theoretically demonstrated that rectification of the ac field of a propagating plasmon, which is enabled by nonlinear terms in Eqs. (1)-(4), yields a spatially dependent dc signal $\delta V(\boldsymbol{r})$. The experimental exploitation of similar nonlinearities has recently led [20] to room-temperature graphene $\mathrm{THz}$ photodetectors. We stress that $\delta V(\boldsymbol{r})$ can be calculated from Eqs. (9) and (10) and can be measured by using lateral probe contacts as in Fig. 1. Simple analytical expressions for $\delta V(\boldsymbol{r})$ have been given in Ref. [38] for the cases $\xi=0,1$. Numerical results for the general case $\xi \neq 0,1$ have been presented in Fig. 3. Such values of dc voltages can be easily measured.

Acknowledgments. We wish to thank R. Hillenbrand, F. Koppens, A. Principi, D. Spirito, A. Tredicucci, and especially M. Lundeberg for many useful discussions. This work was supported by the EC under the Graphene Flagship program (Contract No. CNECT-ICT-604391) and MIUR through the programs "FIRB-Futuro in Ricerca 2010" Project "PLASMOGRAPH" (Grant No. RBFR10M5BT) and "Progetti Premiali 2012" Project “ABNANOTECH."
[1] V. N. Kotov, B. Uchoa, V. M. Pereira, F. Guinea, and A. H. Castro Neto, Rev. Mod. Phys. 84, 1067 (2012).

[2] B. Wunsch, T. Stauber, F. Sols, and F. Guinea, New J. Phys. 8, 318 (2006); E. H. Hwang and S. Das Sarma, Phys. Rev. B 75, 205418 (2007); M. Polini, R. Asgari, G. Borghi, Y. Barlas,
T. Pereg-Barnea, and A. H. MacDonald, ibid. 77, 081411(R) (2008); A. Principi, M. Polini, and G. Vignale, ibid. 80, 075418 (2009); M. Jablan, H. Buljan, and M. Soljačić, ibid. 80, 245435 (2009).

[3] G. F. Giuliani and G. Vignale, Quantum Theory of the Electron Liquid (Cambridge University Press, Cambridge, U.K., 2005). 
[4] S. H. Abedinpour, G. Vignale, A. Principi, M. Polini, W.-K. Tse, and A. H. MacDonald, Phys. Rev. B 84, 045429 (2011); M. Orlita, I. Crassee, C. Faugeras, A. B. Kuzmenko, F. Fromm, M. Ostler, T. Seyller, G. Martinez, M. Polini, and M. Potemski, New J. Phys. 14, 095008 (2012); L. S. Levitov, A. V. Shtyk, and M. V. Feigelman, Phys. Rev. B 88, 235403 (2013).

[5] Z. Fei, G. O. Andreev, W. Bao, L. M. Zhang, A. S. McLeod, C. Wang, M. K. Stewart, Z. Zhao, G. Dominguez, M. Thiemens et al., Nano Lett. 11, 4701 (2011).

[6] Z. Fei, A. S. Rodin, G. O. Andreev, W. Bao, A. S. McLeod, M. Wagner, L. M. Zhang, Z. Zhao, M. Thiemens, G. Dominguez, M. M. Fogler, A. H. Castro Neto, C. N. Lau, F. Keilmann, and D. N. Basov, Nature 487, 82 (2012).

[7] J. Chen, M. Badioli, P. Alonso-González, S. Thongrattanasiri, F. Huth, J. Osmond, M. Spasenovic, A. Centeno, A. Pesquera, P. Godignon, A. Zurutuza Elorza, N. Camara, F. Javier García de Abajo, R. Hillenbrand, and F. H. L. Koppens, Nature 487, 77 (2012).

[8] P. Alonso-González, A. Y. Nikitin, F. Golmar, A. Centeno, A. Pesquera, S. Vélez, J. Chen, G. Navickaite, F. Koppens, A. Zurutuza et al., Science 344, 1369 (2014).

[9] A. Woessner, M. B. Lundeberg, Y. Gao, A. Principi, P. AlonsoGonzález, M. Carrega, K. Watanabe, T. Taniguchi, G. Vignale, M. Polini et al., arXiv:1409.5674.

[10] A. Principi, G. Vignale, M. Carrega, and M. Polini, Phys. Rev. B 88, 195405 (2013).

[11] A. Principi, G. Vignale, M. Carrega, and M. Polini, Phys. Rev. B 88, 121405(R) (2013).

[12] A. Principi, M. Carrega, M. B. Lundeberg, A. Woessner, F. H. L. Koppens, G. Vignale, and M. Polini, Phys. Rev. B 90, 165408 (2014).

[13] A. N. Grigorenko, M. Polini, and K. S. Novoselov, Nat. Photonics 6, 749 (2012); T. Stauber, J. Phys.: Condens. Matter 26, 123201 (2014); T. Low and P. Avouris, ACS Nano 8, 1086 (2014); F. J. García de Abajo, ACS Photonics 1, 135 (2014).

[14] M. Gullans, D. E. Chang, F. H. L. Koppens, F. J. García de Abajo, and M. D. Lukin, Phys. Rev. Lett. 111, 247401 (2013).

[15] F. H. L. Koppens, D. E. Chang, and F. J. García de Abajo, Nano Lett. 11, 3370 (2011).

[16] L. Ju, B. Geng, J. Horng, C. Girit, M. Martin, Z. Hao, H. A. Bechtel, X. Liang, A. Zettl, Y. R. Shen et al., Nat. Nanotechnol. 6, 630 (2011).

[17] H. Yan, X. Li, B. Chandra, G. Tulevski, Y. Wu, M. Freitag, W. Zhu, P. Avouris, and F. Xia, Nat. Nanotechnol. 7, 330 (2012).

[18] V. W. Brar, M. S. Jang, M. Sherrott, J. J. Lopez, and H. A. Atwater, Nano Lett. 13, 2541 (2013).

[19] Z. Fang, Y. Wang, A. E. Schlather, Z. Liu, P. M. Ajayan, F. J. García de Abajo, P. Nordlander, X. Zhu, and N. J. Halas, Nano Lett. 14, 299 (2014).

[20] L. Vicarelli, M. S. Vitiello, D. Coquillat, A. Lombardo, A. C. Ferrari, W. Knap, M. Polini, V. Pellegrini, and A. Tredicucci, Nat. Mater. 11, 865 (2012); D. Spirito, D. Coquillat, S. L. De Bonis, A. Lombardo, M. Bruna, A. C. Ferrari, V. Pellegrini, A. Tredicucci, W. Knap, and M. S. Vitiello, Appl. Phys. Lett. 104, 061111 (2014).

[21] For a recent review, see F. H. L. Koppens, T. Mueller, P. Avouris, A. C. Ferrari, M. S. Vitiello, and M. Polini, Nat. Nanotechnol. 9, 780 (2014).
[22] A. L. Falk, F. H. L. Koppens, C. L. Yu, K. Kang, N. de Leon Snapp, A. V. Akimov, M.-H. Jo, M. D. Lukin, and H. Park, Nat. Phys. 5, 475 (2009).

[23] P. Neutens, P. Van Dorpe, I. De Vlaminck, L. Lagae, and G. Borghs, Nat. Photonics 3, 283 (2009).

[24] See, for example, T. Eberlein, U. Bangert, R. R. Nair, R. Jones, M. Gass, A. L. Bleloch, K. S. Novoselov, A. Geim, and P. R. Briddon, Phys. Rev. B 77, 233406 (2008); Y. Liu, R. F. Willis, K. V. Emtsev, and T. Seyller, ibid. 78, 201403 (2008); R. J. Koch, T. Seyller, and J. A. Schaefer, ibid. 82, 201413 (2010).

[25] L. D. Landau and E. M. Lifshitz, Fluid Mechanics (Pergamon, New York, 1987).

[26] Q. Li and S. Das Sarma, Phys. Rev. B 87, 085406 (2013).

[27] M. Polini and G. Vignale, arXiv:1404.5728.

[28] A. H. Castro Neto, F. Guinea, N. M. R. Peres, K. S. Novoselov, and A. K. Geim, Rev. Mod. Phys. 81, 109 (2009).

[29] W. Wang, P. Apell, and J. Kinaret, Phys. Rev. B 84, 085423 (2011).

[30] A. Y. Nikitin, F. Guinea, F. J. García-Vidal, and L. MartínMoreno, Phys. Rev. B 84, 161407(R) (2011).

[31] J. Christensen, A. Manjavacas, S. Thongrattanasiri, F. H L. Koppens, and F. J. García de Abajo, ACS Nano 6, 431 (2012).

[32] W. Wang and J. M. Kinaret, Phys. Rev. B 87, 195424 (2013).

[33] A. Principi, G. Vignale, M. Carrega, and M. Polini (unpublished).

[34] S. Selberherr, Analysis and Simulation of Semiconductor Devices (Springer, Berlin, 1984); D. Vasileska and S. M. Goodnick, Computational Electronics (Morgan \& Claypool, San Rafael, CA, 2006).

[35] A. Tomadin and M. Polini, Phys. Rev. B 88, 205426 (2013).

[36] D. Svintsov, V. Vyurkov, V. Ryzhii, and T. Otsuji, Phys. Rev. B 88, 245444 (2013).

[37] We have also solved the problem numerically with the full nonlocal relation $\Phi(\boldsymbol{r}, t)=\int d^{2} \boldsymbol{r}^{\prime} \varphi\left(\left|\boldsymbol{r}-\boldsymbol{r}^{\prime}\right|\right) \delta n\left(\boldsymbol{r}^{\prime}, t\right)$, where $\varphi(\mid \boldsymbol{r}-$ $\left.\boldsymbol{r}^{\prime} \mid\right)$ is the 2D Fourier transform of the electrostatic potential [35] $\varphi_{k}=-2 \pi e[1-\exp (-2 k d)] /\left\{\bar{\epsilon} k\left[1-\epsilon_{\mathrm{d}} \exp (-2 k d) / \epsilon_{\mathrm{s}}\right]\right\}$, where $\epsilon_{\mathrm{s}, \mathrm{d}} \equiv \epsilon_{1} \pm \epsilon_{2}$ and $\bar{\epsilon}=\epsilon_{\mathrm{s}} / 2$. In the limit $k d \rightarrow 0, \varphi_{k} \rightarrow$ $-e / C, \varphi\left(\left|\boldsymbol{r}-\boldsymbol{r}^{\prime}\right|\right) \rightarrow-e \delta\left(\boldsymbol{r}-\boldsymbol{r}^{\prime}\right) / C$, and one recovers Eq. (4). No appreciable differences have been noticed for realistic parameters.

[38] See Supplemental Material at http://link.aps.org/supplemental/ 10.1103/PhysRevB.91.081402 where we discuss the boundary conditions we used, detail the perturbative solution of the hydrodynamic equations, explain the calculation of the plasmon spectrum, and present analytical results for the rectified signal in simple cases.

[39] A. Tomadin, G. Vignale, and M. Polini, Phys. Rev. Lett. 113, 235901 (2014)

[40] S. Thongrattanasiri, I. Silveiro, and F. J. García de Abajo, Appl. Phys. Lett. 100, 201105 (2012).

[41] The quantity $\delta n$ can be estimated as follows. Let us assume that a laser with power $P=20 \mathrm{~mW}$, concentrated on a spot size with diameter $d=20 \mu \mathrm{m}$, is used to launch plasmons, as in typical midinfrared experiments [9]. We can estimate the electric field in the 2D electron system underneath the tip apex as (switching to SI units for the sake of convenience) $E \sim \sqrt{\kappa \delta 8 P Z_{0} /\left(\pi d^{2}\right)}$, where $Z_{0}$ is the vacuum impedance. The factor $\delta$ measures the fraction of power funneled by the tip into the electronic subsystem, while the factor $\kappa$ is related to 
the electric-field enhancement due to the tip apex. The electron density created by $E$ can be estimated from linear response theory [3], $\delta n \sim k \sigma E /(e \omega)$, where $\sigma=\sigma(k, \omega)$ is the 2D MDF conductivity at wave vector $k$ and frequency $\omega$. For $\delta=0.1$ and $\kappa=10$ we find $\delta n \sim 5 \times 10^{9} \mathrm{~cm}^{-2}$. The estimate $\delta n / \bar{n}=1 \%$ for an average carrier density $\bar{n} \sim 10^{12} \mathrm{~cm}^{-2}$ in the THz follows from an increase of $d$ from 20 to $100 \mu \mathrm{m}$, keeping fixed $P, \delta$, and $\kappa$.

[42] M. Dyakonov and M. Shur, IEEE Trans. Electron Devices 43, 380 (1996). 